\title{
Adaptación y percepción de estudiantes extranjeros respecto a la cultura y el sistema educativo en una universidad pública en Colombia
}

\author{
Adaptation and perception of foreign students about culture and education \\ system at a Public University in Colombia
}

Michael Hernández Bolívar ${ }^{1}$ Yesid José Ortega Pacheco ${ }^{2}$

\section{Resumen}

El objetivo principal del presente estudio fue describir el proceso de adaptación y percepción de los estudiantes universitarios extranjeros respecto a la cultura y el sistema educativo en una universidad pública en Colombia. Se realizó un estudio cualitativo en una muestra compuesta por (7) siete sujetos provenientes de México, Brasil, Argentina y Francia. Se encontró que estos procesos no tienen finalización exacta o permanente, teniendo en cuenta que la sociedad, la cultura, el modelo educativo y la universidad están en cambio constante. Por tal motivo, los estudiantes debieron adaptarse, aprendiendo de las experiencias y de lo que los otros pudieran aportarles. Así mismo, mostraron ser permeables a las diferentes problemáticas sociales que enfrenta su nuevo contexto, llegado a comprenderlas e intentar dar una explicación basados en sus propias percepciones.

Palabras clave: Adaptación; percepción; aculturación; estudiantes extranjeros.

\section{Abstract}

This research paper aimed to describe the adaptation and perception process of foreign students in relation to the culture and the education system at a Public University in Colombia. A qualitative study was carried out with a sample of (7) seven students from Mexico, Brazil, Argentina and France. It was found that these processes do not have an exact or permanent ending, taking into account that society, culture, educational model and the university are constantly changing. Consequently, the students had to adapt by learning from the experiences and what others could bring to them. Likewise, students showed to be permeable to the different social problems they might face in the new context, understanding and trying to give an explanation to it, based on their own perceptions.

\footnotetext{
Psicólogo. Especialista en Gestión de Procesos Psicosociales. Fundación Fe y Alegría. Correo: michaelhdez@gmail.com. Orcid: https://orcid.org/oooo-0003-4328-1693

2 Psicólogo. Fundaprobic. Correo: Yesid.1594@gmail.com. Orcid: https://orcid.org/oooo-0oo2-7274-0150
}

Recibido: 24/04/2019 Aprobado: 09/05/2019 
Key Words: Adaptation; perception; acculturation; foreign students.

\section{Introducción}

En la actualidad, la mayoría de instituciones de educación superior públicas se encuentran en proceso de acreditación por alta calidad, motivo por el cual han procurado mejorar el servicio que ofrecen y fortalecer el impacto de sus egresados no sólo a nivel regional sino también en todo el país (Hernández, Arcos y Sevilla, 2013).

Esto ha conllevado a que se generen dentro de las universidades convenios internacionales que permiten a diferentes estudiantes extranjeros realizar intercambios académicos en instituciones colombianas propiciando el enriquecimiento educativo y cultural. Esta medida no sólo funciona como estrategia administrativa, sino que también impacta en cómo se proyectan a nivel social las universidades públicas (De Wit, Gacel-Ávila y Knobel. 2017). Por lo tanto, las investigaciones recientes en materia de educación han mostrado gran interés en comprender cómo es el proceso que viven estos nuevos estudiantes en la universidad y cómo afecta su calidad de vida al verse reflejado el contraste cultural del país que los acoge al de procedencia (Sosa y Zubieta, 2015).

\section{Revisión de literatura}

El proceso de adaptación sociocultural está vinculado con el aprendizaje de tipo social, por tal motivo, se relaciona con el tiempo de estadía, las relaciones interpersonales y la distancia cultural, llegando a determinar notablemente el proceso de adaptación a una nueva sociedad (Basabe, Zoblina y Paez, 2004). Los estudiantes al llegar por primera vez a una metrópolis desconocida se encuentran sumergidos automáticamente en todas las normas, costumbres, valores y creencias que esta posee, es por esto que aprenden a desenvolverse en un ambiente cultural nuevo (Ramírez, 2017).

El proceso de adaptación no sólo se produce a nivel físico y cultural, también a nivel psicológico, dado que se genera un cambio total en los estilos y hábitos de vida que le permiten a los nuevos estudiantes obtener mayor desenvolvimiento en la ciudad donde son recibidos (Benatuil y Laurito, 2009; Pérez-Pulido, 2016).

Por otra parte, la percepción juega un papel fundamental en este proceso, ya que mediante esta los estudiantes extranjeros extraen y seleccionan del contexto la información que resulta relevante. Además, propicia un estado de claridad y lucidez de la realidad del lugar donde se encuentran (Gonzáles, 2006; Pardo, Franco y Pardo 2016). En consecuencia, los nuevos estudiantes tienen una visión holística de cómo es el funcionamiento institucional, el cual les permitirá ser agente activo en su programa, entender más a fondo la cultura y los diferentes factores que esta trae consigo, tales como las jergas, las comidas típicas y los medios de transporte que son necesarios 


\section{EDUCACIÓN}

para la convivencia y la adaptación. Por consiguiente, no debe abordarse de manera aislada sino como factor complemento al proceso de adaptación, teniendo en cuenta que el estudiante de intercambio estará inmerso en un nuevo mundo en el cual las personas que conviven en el juegan un papel precipitante teniendo en cuenta que a futuro determinará si realmente logran adaptarse o por el contrario, no consiguen satisfactoriamente lo esperado en el intercambio estudiantil (Berry, 2003; Castro, 2011).

Desde la psicología intercultural, uno de los principales referentes en esta temática es Berry (1989) con su modelo de aculturación donde destaca cuatro componentes de la adaptación. El primero de ellos se conoce como asimilación, donde el inmigrante adquiere las características de la nueva cultura, si este llega a identificarse con sus propios valores y los de la nueva cultura, se conoce como integración. El tercero es la segregación, es decir, el sujeto no desea aceptar las características de la cultura que lo recibe y prefiere continuar reforzando su cultura de origen, y, por último, la marginalización se produce cuando la persona pierde por completo sus características sociales precedentes, pero tampoco practica las de la nueva sociedad (Berry, 2005).

Según González y Flórez (2011) la nueva adaptación que realizan los estudiantes internacionales debe ser lo más rápido posible, esto con la finalidad que el individuo pueda sentirse cómodo frente al nuevo eslabón social al que pertenecerá. Para ello, es necesario un engranaje que incorpore las diferencias e igualdades entre la cultura de procedencia y la receptora, lo cual, según estos autores, en muchos casos provoca la extinción de ciertas características de la cultura propia.

Pero más allá, son estos procesos los que le dan una presentación y representación a nivel mundial de cómo es Colombia y en su defecto, cómo es su cultura y su sistema educativo, generando en ellos ideas y/o percepciones generales que luego se extrapolan al lugar de procedencia ya sean buenas o malas y que representan al país en el exterior.

\section{Materiales y métodos}

La presente investigación se realizó desde el paradigma cualitativo, el cual estudia la realidad en su propio contexto, se orienta a comprender y ahondar en los fenómenos, profundizando desde la percepción de los participantes, tratando de dar sentido a los significados que estos tienen para los involucrados (Hernández, Fernández y Baptista, 2010). El diseño empleado fue de tipo fenomenológico donde se vislumbra el significado que los individuos dan a su experiencia. El propósito de la fenomenología es intentar comprender el proceso de interpretación subjetiva de cada sujeto y a partir de allí establecer hipótesis del porqué de los comportamientos e ideologías de las personas (Sandoval, 2002). 


\section{Población}

Los sujetos que hicieron parte del estudio fueron (7) siete estudiantes extranjeros, que realizaron un semestre de intercambio académico, provenientes de México, Brasil, Argentina y Francia. Estos fueron seleccionados a través del muestreo no probabilístico por conveniencia, donde estos son escogidos a criterios del investigador por las características de proximidad y accesibilidad para cumplir con el objetivo de investigación (Salkind, 1998; Otzen y Manterola, 2017).

\section{Instrumento}

Respecto al proceso de recolección de la información, la técnica empleada fue la entrevista semi estructurada, la cual consta de una serie de preguntas previamente elaboradas por los investigadores que son presentadas a los sujetos de estudio; sin embargo, al ser abierta permite que en el transcurso de la misma puedan surgir otro tipo de cuestionamientos que sirvan como complemento de la investigación con la finalidad de ampliar la información y contribuir a la consecución del objetivo de estudio (Montañés, 2009).

\section{Análisis de datos}

Se utilizó el análisis de contenido como herramienta de interpretación te textos escritos (Bernete, 2013) y el software Atlas.ti para la comprensión de los datos suministrados por los participantes, permitiendo así la codificación, categorización y el respectivo análisis de los datos encontrados (Varguillas, 2006; San Martín, 2014).

\section{Resultados y discusión}

Tabla No. 1: Presentación de categorías, subcategorías y códigos

\begin{tabular}{|c|c|c|}
\hline Categorías & Subcategorías & Códigos \\
\hline \multirow{2}{*}{ Adaptación } & Social & Relaciones sociales \\
\cline { 2 - 3 } & Intercambio & Expectativas, motivación \\
\hline \multirow{2}{*}{ Percepción } & Intereses & $\begin{array}{c}\text { Sistema educativo, cultura, infraes- } \\
\text { tructura }\end{array}$ \\
\cline { 2 - 3 } & Opiniones & Negativas, positivas \\
\hline
\end{tabular}

Fuente: Elaboración propia con base en el discurso de las participantes.

A partir de la narrativa de los estudiantes extranjeros, se elaboraron dos categorías principales, la primera conocida como Adaptación y la segunda como Percepción, cada una de ellas con sus respectivas subcategorías y códigos. 


\section{EDUCACIÓN}

La categoría Adaptación está conformada por las subcategorías: Social e intercambio. La primera compuesta por el código: Relaciones sociales, donde los entrevistados aludieron:

(...) pues en un principio si nos sentíamos como rechazados... bueno a lo mejor, nos tenemos nosotros que acercar y estuvimos utilizando diferentes estrategias porque nos costó trabajo (SC1.4).

Como curso con personas de diferentes clases, se me ha hecho difícil y no me permite establecer amistad (SC1.3).

Por otra parte, la subcategoría: Intercambio engloba el código: Expectativas, en este los estudiantes manifestaron:

(...) era más conveniente ir a estudiarlo a un país donde es similar a México que a ir a un lugar donde se es más avanzado y regresar a mi país con ideas bastante novedosas ( $\left.\mathrm{SC}_{1.1}\right)$.

Tenía una imagen idealizada de Colombia porque en mi país recibimos cosas de ustedes. Es muy diversa y uno no se alcanza a imaginar los regionalismos, la diversidad cultural (...) (SC1.2).

Subsiguientemente, en el código; Motivación, los estudiantes extranjeros expresaron:

Yo vine acá por el intercambio, además de conocer el país, la cultura, la diversidad, me parece un país muy bonito ( $\left.\mathrm{SC}_{1} .6\right)$.

Vine al país por mi intercambio, además que México tiene buenas relaciones con este país y conocer personas nuevas (...)(SC1.5).

Dentro de la categoría: Percepción, se encuentran las subcategorías: Intereses y Opiniones. La primera de ellas está conformada por los códigos: Sistema educativo, Cultural e Infraestructura. Con relación al Sistema educativo, los entrevistados enunciaron:

(...) la buena capacitación de algunos de los maestros, las clases, la suficiente infraestructura física y la calidad académica es buena (...) (SC2.1).

El sistema educativo es bueno, tiene competitividad, los pibes con los que hemos convivido se ve que tienen conocimientos generales ( $\mathrm{SC}_{2.4}$ ). 
Aunque se encontró discrepancia por parte de algunos de los entrevistados, los cuales manifiestan no sentirse acorde con el sistema:

Lamento el alto costo que se tiene que pagar para poder acceder a la educación, la cual en muchos otros países es gratuita(SC2.2).

La educación allá siento que nos hacen más autónomos... en cuanto al nivel si en mi U el nivel es mucho más alto, aquí las exigencias de algunos son como muy mínimas, de igual los profesores cuando dicen su preparación es muy baja en cuanto a los de allá (SC2.5).

Por parte del código: Cultura, los educandos exteriorizaron:

La cultura es muy alegre, pero sobre todo muy marcada por el miedo que se sembró en la época de los años 9o, el cual según ellos afecta a la libre expresión y le impide a la gente luchar por sus derechos" (SC2.1).

"Esta cultura tiene mucho que ofrecer (...) (SC2.7).

Yo quería conocer, he leído mucho sobre los conflictos, pero no me daba miedo venir y encontrar lo que todo mundo dice sobre las drogas y la guerrilla... eso aquí hace parte de la cultura (SC2.6).

Posteriormente, luego de conocer y descubrir la auténtica cultura colombiana, los estudiantes se dan cuenta que es totalmente diferente a lo que se dice del país en el exterior:

Colombia es un país totalmente diferente a lo cual se conoce o se dice en el exterior $\left(\mathrm{SC}_{2.4)}\right.$.

Con relación al código Infraestructura, los entrevistados expresaron lo siguiente: En cuanto a la estructura, se me hace muy bonita, tiene mucho verde para la frescura y eso ayuda para el calor de acá... (SC2.5).

El internet en diferentes zonas eso es un avance, los salones tiene equipos multimedia, tecnológicamente están bien, a diferencia de México (SC2.3).

La universidad es muy bonita, además que tiene una infraestructura muy grande y les ofrece a los estudiantes mucho (SC2.2). 


\section{EDUCACIÓN}

La segunda subcategoría: Opiniones, compuesta por el código: Positivo, los entrevistados manifestaron sentires a gusto con la biodiversidad cultural, biológica, étnica y política que cuenta la cultura colombiana, además de la solidaridad de la gente, es por esto que refieren:

Aquí todos, yo veo que todos se saludan, el ambiente es más cálido con las personas (SC2.3).

Igualmente hicieron mención sobre el sistema educativo planteando la calidad académica universitaria:

La metodología nos ha parecido buena; sin embargo aún les falta, pero sabemos que están en proceso de avance educativo (SC2.6).

Por otra parte, en paralelo con el código: Negativo, los entrevistados manifestaron diversos aspectos que según sus percepciones deberían mejorar o cambiar, entre eso:

La biblioteca igual, se me hace que está muy pobre en cuanto a materiales porque es muy pequeña y siento que aquí hay muchas carreras y hace falta bastante material bibliográfico (SC2.1).

Además, hicieron mención sobre su opinión acerca de la calidad de los estudiantes universitarios y los costos de la educación:

Los alumnos deben ser más críticos y autónomos (SC2.4).

La universidad es semi-pública, si porque las cuotas comparadas a lo que pagamos, se nos hace que es bastante (SC2.2).

Basados en la categoría: Adaptación, se observa cómo los estudiantes extranjeros lograron implementar estrategias y métodos de aculturación para poder adaptarse al nuevo contexto y por ende, sobrevivir a este. Uno de los factores principales que contribuyó en este proceso fueron las habilidades comunicativas, ya que, los estudiantes utilizaron tácticas para la supervivencia al medio con el fin de instaurar nuevas formas de actuar para mejorar las relaciones interpersonales. Además, adoptaron la cultura social del país de destino como método de socialización con los pares y la adaptación al nuevo sistema educativo (Benatuil y Laurito, 2009; Pérez-Pulido, 2016).

Por otra parte, hacen mención de la importancia de escoger un país como Colombia para efectuar su programa de intercambio, puesto que reconocen lo que este a nivel social y cultural puede aportarles a su formación personal y profesional. Con relación a lo anterior, Castro (2011) da a conocer de manera explícita que muchos de los estudiantes extranjeros que realizan procesos de intercambio estudiantil prefieren hacerlo 
en países que le puedan aportar a su formación profesional bases claras para afrontar cualquier situación nunca antes vivida. Se destaca que, a pesar de las dificultades en el proceso de adaptación, los estudiantes reconocen que fueron estas las que les permitieron aprender y mejorar las estrategias que erróneamente habían empleado, lo cual les ayudo a poder mantenerse y resistir a las exigencias del nuevo entorno y con ello lograr superarlas.

De acuerdo con la categoría: Percepción, los estudiantes manifestaron tener una concepción errónea de Colombia como resultado de las diferentes opiniones e información recibida por parte de los medios de comunicación de su país, de tal modo, este estudio demuestra que las expectativas de los extranjeros estuvieron encaminadas en todas aquellas riquezas sociales y culturales que Colombia les podría brindar. Además, la posibilidad de entrar en contacto con una sociedad estigmatizada alrededor del mundo por las múltiples situaciones de conflicto que ha vivido el país y que les ayudaría a crecer a nivel personal y profesional (Carvajal, 2011).

Los entrevistados expresaron sus opiniones respecto al sistema educativo haciendo mención a la privatización de la educación y la manera en cómo se llevan a cabo el proceso de aprendizaje y enseñanza en la universidad pública, mostrando que todavía al Sistema de Educación Superior en Colombia le hace falta seguir fortaleciéndose en busca del reconocimiento y competitividad a nivel internacional. Según Castaño-Duque y García-Serna (2012), las problemáticas educativas en cuanto a calidad también se han visto afectados por la poca inversión financiera. Es por esto que los estudiantes establecieron paralelos en cuanto a la calidad educativa que poseen y que imparten las universidades donde proviene a la que posee a nivel general Colombia.

\section{Conclusiones}

A través de los datos recogidos se concluye que los estudiantes extranjeros se encuentran satisfechos respecto a su proceso de intercambio estudiantil en la Universidad y reiteran su motivación en quedarse mucho más tiempo y continuar disfrutando de la cultura y aprender de las costumbres colombianas, dado que consideran que el país cuenta con los recursos necesarios para brindarles buenas bases para favorecer su proceso de adaptación a un sistema que es totalmente diferente para ellos.

También se encontró que los estudiantes presentan permeabilidad frente a los problemas que atraviesa la sociedad y a nivel macro la cultura colombiana, destacando aspectos como el bajo nivel educativo de la educación superior, el conflicto social que atraviesa el país y la ideología de pensamiento por parte de los habitantes. En cuanto a la cultura local, se evidenció por parte de los entrevistados, muchas críticas tales como los malos manejos de basura y la falta de aprovechamiento de los recursos con los se cuenta. 


\section{EDUCACIÓN}

A partir de los testimonios de los entrevistados, se hace mención que el país tiene diferentes visiones alrededor del mundo tanto positivas como negativas en temas como el narcotráfico, la guerrilla, los problemas políticos que afectan a las sociedades y la economía; en aspectos positivos aluden la gran biodiversidad cultural, la calidad humana y la geografía del país.

Por tal motivo, se concluye que el proceso de adaptabilidad y percepción de los estudiantes universitarios extranjeros respecto a la cultura y sistema educativo, no tiene una finalización exacta o permanente, ya que, la sociedad, cultura y universidad está en cambio constante a lo cual estos deben adaptarse cada día construyendo o aprendiendo de los mismos y para los mismos.

\section{Agradecimiento}

Esta publicación obtuvo el financiamiento de: El Fondo de Asistencia Internacional de los Estudiantes y Académicos Noruegos, (SAIH).

\section{Lista de referencias}

Basabe, N., Zlobina, A. y Páez, D. (2004). Integración socio-cultural y adaptación psicológica de los inmigrantes en el País Vasco. Servicio Central de Publicaciones del Gobierno Vasco: Vitoria-Gasteiz.

Benatuil, D. y Laurito, J. (2009). La adaptación cultural en estudiantes extranjeros. Psicodebate: Psicología, Cultura y Sociedad, 10, 119-134.

Bernete, F. (2013). Análisis de contenido. En A.L. Marín y A. Noboa (Coords.), Conocer lo social: estrategias y técnicas de construcción y análisis de datos (pp. 222-262). España: Universidad Complutense de Madrid.

Berry, J.W. (1989). Acculturation et adaptation psychologique. In: J. Retschitzky, M. BosselLagos, P. Dasen (Eds.), La Recherche interculturelle, t. I. Paris: L'Harmattan.

Berry, J.W. (2003). Conceptual approaches to acculturation. En K. Chun, P. BallsOrganista \& G. Margin (Eds.), Acculturation: Advances in theory, measurement and applied research (pp. 17-37). Washington. DC: APA Press.

Berry, J.W. (2005). Acculturation: Living successfully in two cultures. International Journal of Intercultural Relations, 29(6), 697-712. 
Carvajal, L. A. (2014). Análisis de la gestión de competitividad en Colombia como destino turístico. Revista EAN, (76), 78-90.

Castaño-Duque, G. A. y García-Serna, L. (2012). Una revisión teórica de la calidad de la educación superior en el contexto colombiano. Educ. Educ, 15(2), 219-243.

Castro, A. (2011). Estrategias de aculturación y adaptación psicológica y sociocultural de estudiantes extranjeros en la Argentina. Interdisciplinaria, 28(1), 115-130.

De Wit, H., Gacel-Ávila, J. y Knobel, M. (2017). Estado del arte de la internacionalización de la educación superior en América Latina. Revista de Educación Superior en América Latina, (2). DOI: http://dx.doi.org/10.14482/esal.2.10151

Gonzáles, J. M. (2006). Perspectivas contempératenos cobre la cognición, percepción, categorización y conceptualización. México: Siglo XXI editores, s.a de c.v. en coedición con la Universidad Autónoma del estado de Morelos.

Gonzáles, M. E. y Flórez, M. P. (2011). Cambios culturales: Percepciones de estudiantes universitarios extranjeros en su adaptación a la cultura mexicana. Observatorio (OBS*) Journal, 5, (3), 133-155. DOI: https://doi.org/10.15847/obsOBS532011459

Hernández, R., Fernández, C. y Baptista, P. (2010). Metodología de la investigación. México: Edit. McGraw-Hill.

Hernández, G., Arcos, J. L. y Sevilla, J. J. (2013). Gestión de la calidad bajo la Norma ISO 9001 en instituciones públicas de educación superior en México. Calidad en la educación, (39), 81-115.

Montanés, M. (2009). Metodología y técnica participativa. Teoría y práctica de una estrategia de investigación participativa. España: Editorial UOC.

Otzen, T. y Manterola, C. (2017). Técnicas de muestro sobre una población a estudio. International Journal of Morphology, 35(1), 227-232. DOI: http://dx.doi. org/10.4067/So717-95022017000100037

Sosa, F. M. y Zubieta, E. (2015). La experiencia de migración y adaptación sociocultural: identidad, contacto y apoyo social en estudiantes universitarios migrantes. Psicogente, 18(33), 36-51. 
Pardo, I., Franco, N. F. y Pardo, A. C. (2016). Percepción de la pasantía en promoción de la salud como estrategia de la atención primaria en salud. Revista Cubana de Medicina General Integral, 35(4).

Pérez-Pulido, I. (2016). El proceso de adaptación de los estudiantes a la universidad en el Centro Universitario de Los Altos de la Universidad de Guadalajara (Tesis Doctoral). Instituto Tecnológico y de Estudios Superiores de Occidente, Tlaquepaque, Jalisco.

Ramírez, M. P. (2017). El proceso de adaptación sociocultural: un modelo sociocognitivo de aculturación (Tesis de Doctorado). Universidad Pontificia Comillas ICAI-ICADE, Madrid, España.

Salkind, N. J. (1998). Métodos de investigación. México: Prentice Hall.

Sandoval, C. A. (2002). Investigación cualitativa. Colombia: ARFO editores Ltda.

San Martín, D. (2014). Teoría fundamentada y Atlas.ti: recursos metodológicos para la investigación educativa. Revista electrónica de investigación educativa, 16(1), 104-122.

Varguillas, C. (2006). El uso de atlas.ti y la creatividad del investigador en el análisis cualitativo de contenido upel. Instituto pedagógico rural el mácaro. Revista de educación, 12 (2), 73-87. 